Renato PAsSini JúNIOR ${ }^{1}$

Marcelo Luís Nomura ${ }^{2}$

Gabriel Tilu Politano ${ }^{3}$

Revisão

\section{Palavras-chaves}

Periodontite

Doenças periodontais

Citocinas

Complicações na gravidez/

fisiopatologia

Saúde bucal

Gestantes

Medição de risco

Keywords

Periodontitis

Periodontal diseases

Cytokines

Pregnancy complications/

physiopathology

Oral health

Pregnant women

Risk assessment

\title{
Doença periodontal e complicações obstétricas: há relação de risco?
}

\author{
Periodontal disease and obstetrical complications: \\ is there a risk relationship?
}

\section{Resumo}

Estudos têm apontado possíveis relações de risco existentes entre doenças bucais, principalmente a doença periodontal, e complicações gestacionais, como parto prematuro, nascimento de recém-nascidos de baixo peso e pré-eclâmpsia. As explicações para tais hipóteses baseiam-se no fato de a doença periodontal ser de origem infecciosa, o que poderia provocar aumento de citocinas inflamatórias no sangue materno, por liberação direta da bolsa periodontal ou por disseminação de bactérias patogênicas, induzindo sua produção sistêmica. Esta suposição fundamenta-se no conhecimento de que a fisiopatologia das complicações obstétricas citadas está associada à presença de algumas citocinas no sangue materno. $\bigcirc$ presente trabalho teve como objetivo realizar revisão da literatura em busca de evidências para estas supostas associações. Apesar do grande número de estudos clínicos encontrados nesta revisão, observa-se a falta de padronização metodológica dos mesmos, fato que limita conclusões definitivas a respeito. Por outro lado, o fato de a doença periodontal ainda não ser comprovadamente um fator de risco para as complicações obstétricas não diminui a importância da manutenção da saúde bucal das gestantes, que devem apresentar condições orais que propiciem adequada alimentação, sem dor e sangramento, e assim manter seu aporte nutricional adequado.

\section{Abstract}

Studies have shown possible risk relations among oral illnesses, mainly periodontal disease and adverse pregnancy outcomes, such as prematurity, low birth weight and preeclampsia. The explanation for this hypothesis is based on the fact that periodontal disease is an infectious state, which may increase maternal serum cytokines through the release of such agents directly from the periodontal pocket or by through the dissemination of pathogenic bacteria, inducing systemic production. This assumption is based on the knowledge that the physiopathology of the pregnancy complications cited above is associated with the presence of some cytokines in the maternal serum. The present study work has the objective to review literature in search of evidence to these alleged associations. Although a number of clinical studies have been found in this review, we noticed a lack of methodological standards, what limits the conclusions about this topic. On the other side, the fact that periodontal disease is not yet a confirmed risk factor for adverse pregnancy outcomes does not reduce the importance of oral health maintenance during pregnancy, since it is important to allow adequate feeding without pain and bleeding in order to maintain an adequate nutritional supply.

Correspondência:

Renato Passini Júnior Departamento de Tocoginecologia da FCM/UNICAMP Rua Alexander Fleming, 101 - Cidade Universitória - Barão Geraldo CEP 13083881 - Campinas/SP Fones: (19) $3521-9304 /$ (19) $3521-9335$ Fax: (19) 3521-9304 E-mail: passini@caism.unicamp.br

Recebido 29/05/2007

Aceito com modificacōes
Departamento de Tocoginecologia da Faculdade de Ciências Médicas da Universidade Estadual de Campinas - UNICAMP Campinas (SP), Brasil.

Doutor, Professor Assistente do Departamento de Tocoginecologia da Faculdade de Ciências Médicas da Universidade Estadual de Campinas - UNICAMP - Campinas (SP), Brasil.

2 Doutor, Médico do Departamento de Tocoginecologia da Faculdade de Ciências Médicas da Universidade Estadual de Campinas - UNICAMP - Campinas (SP), Brasil.

${ }^{3}$ Pós-graduando do Departamento de Tocoginecologia da Faculdade de Ciências Médicas da Universidade Estadual de Campinas - UNICAMP - Campinas (SP), Brasil. 


\section{Introdução}

A gestação é um momento em que a saúde bucal deve ser acompanhada com muito cuidado, já que, neste período, algumas alterações mórbidas podem se tornar mais prevalentes. Anormalidades como hiperemia, edema e grande tendência ao sangramento gengival têm sido classificadas como gengivite gravídica ${ }^{1}$. A prevalência dessa alteração varia entre 35 e 100\%, tendo sua severidade gradualmente aumentada até a $36^{a}$ semana de gestação ${ }^{2}$.

É comumente aceita a teoria de que o brusco aumento dos hormônios femininos circulantes durante a gestação é responsável pela exacerbação da reação inflamatória gengival, principalmente por sua ação vasodilatadora ${ }^{3}$. Apesar de a gestação intensificar a reação inflamatória no tecido gengival, o biofilme dentário - acúmulo de bactérias no dente -, é de fundamental importância para o desenvolvimento desta afecção, sendo que seu controle por meio de escovação apropriada parece evitar inflamação e sangramento ${ }^{4}$.

A necessidade dos cuidados bucais durante a gestação baseia-se em dois motivos principais: as gestantes devem se alimentar corretamente e, por isso, não seria admissível que apresentassem dor e/ou mobilidade dentária, e infecções periodontais poderiam se disseminar pela corrente sanguínea e estimular a produção de citocinas inflamatórias. Em decorrência das atuais publicações que relacionam algumas importantes complicações gestacionais (parto prematuro, recém-nascidos de baixo peso e pré-eclâmpsia) com a presença de citocinas também produzidas no periodonto infeccionado, esta revisão buscou avaliar se a literatura disponível permite suportar tal relação de risco.

\section{Citocinas inflamatórias periodontais}

\section{e sua disseminação sistêmica}

A doença periodontal é uma das infecções bucais mais prevalentes nos seres humanos, sendo caracterizada por inflamação e sangramento gengival. Quando os agentes causadores desta afecção não são removidos periodicamente, tendem a atingir o tecido de suporte dos dentes, o osso alveolar, e estimular sua reabsorção por meio de reação inflamatória .

Nas bolsas periodontais podem ser isoladas diversas espécies bacterianas, muitas delas gram-negativas e algumas se caracterizando por alta patogenicidade ${ }^{6}$.

Quando o organismo humano reconhece a presença destas bactérias, inicia-se a reação de imunidade inata, primeira linha de defesa contra os agentes agressores. Além da atuação dos macrófagos fagocitários, há a liberação de algumas citocinas inflamatórias, proteínas que regulam e coordenam muitas das atividades das células responsáveis pela imunidade inata ${ }^{7}$.

Diversas são as citocinas inflamatórias encontradas na bolsa periodontal, destacando-se a interleucina (IL) 1-beta, prostaglandina $\mathrm{E}_{2}^{8}$, IL-6 e o fator de necrose tumoral alfa (TNF-alfa) .

Se estas citocinas, ou mesmo os agentes infectantes, permanecessem exclusivamente no interior da bolsa periodontal, sem atingir a corrente sanguínea, não se justificariam preocupações buscando relações entre doença periodontal e alterações sistêmicas. No entanto, sabe-se que esta disseminação pode ocorrer, sendo o exemplo mais conhecido o risco de desenvolvimento de endocardite bacteriana após procedimentos cirúrgicos bucais em pacientes com algumas alterações cardíacas, o que levou a American Heart Association a preconizar a profilaxia antibiótica para estes casos ${ }^{10}$.

Em relação às citocinas, sabe-se que seus níveis sanguíneos podem estar aumentados em pacientes com a doença periodontal, sugerindo sua disseminação a partir destes focos. Há evidências de que, imediatamente após o tratamento periodontal, ocorre elevação dos níveis séricos de IL-6 e TNF-alfa ${ }^{11}$. Este aumento poderia ser explicado de duas maneiras: pela disseminação das citocinas durante a raspagem do tecido infectado ou pela ação circulante das próprias bactérias, induzindo produção das citocinas sistemicamente.

\section{Doença periodontal e as complicações gestacionais}

Baseando-se na comprovação da disseminação sanguínea das citocinas e/ou bactérias provenientes da infecção periodontal, acredita-se haver associação entre esta afecção e o aumento do risco de algumas alterações sistêmicas, principalmente diabetes mellitus ${ }^{12}$ e alterações cardiovasculares ${ }^{6}$.

Pode-se sugerir, também, a relação entre a doença bucal e outras alterações, que estão comprovadamente relacionadas com a presença e aumento destas mesmas citocinas, como parto prematuro ${ }^{13}$, recém-nascidos de baixo peso ${ }^{14}$ e pré-eclâmpsia ${ }^{15-19}$. Diversos tipos de estudos são desenhados para buscar estas relações de risco; no entanto, conforme discutido adiante, há fortes restrições nas conclusões destas pesquisas.

\section{Parto prematuro e recém-nascidos de baixo peso}

Em 1996, um estudo do tipo caso-controle com 124 gestantes foi realizado para avaliar se a doença periodontal poderia ser considerada fator de risco para nascimento de prematuros ou de recém-nascidos com baixo peso $(<2.500 \mathrm{~g})^{14}$. Para diminuir a influência dos fatores de risco conhecidos associados (cigarro, álcool, drogas, infecções geniturinárias, cuidado pré-natal, 
paridade, dentre outros), os autores usaram métodos estatísticos para evitar a interferência de variáveis confundidoras. Todas as gestantes tiveram o periodonto avaliado, sendo que aquelas do grupo de casos apresentaram piores condições periodontais que as do grupo controle. Tal achado foi significativo, com razão de chances de 7,9.

Esta pesquisa pioneira estimulou os pesquisadores dessa área a estudar as relações entre doença periodontal, prematuridade e outras complicações gestacionais. Atualmente, centenas de trabalhos já foram publicados neste sentido, mas os resultados persistem conflitantes ${ }^{20}$.

Sugere-se que infecções podem ser consideradas um dos fatores etiológicos do parto pré-termo espontâneo ${ }^{21,22}$. Mesmo que a literatura considere como principais infecções relacionadas a esta complicação gestacional aquelas da região geniturinária, não se deve desmerecer sítios infecciosos em outros locais do organismo.

Focos infecciosos em atividade, independente da relação topográfica, têm potencial de acometer outras regiões, principalmente pela corrente sangüínea ${ }^{10,23}$, mas, mesmo assim, ainda não se tem certeza de que as bactérias presentes em infecções periodontais são realmente perigosas para a gestação.

Com objetivo de resolver esta dúvida, alguns autores realizaram pesquisa em que injetaram os lipopolissacarídeos das principais bactérias periodontopatogênicas (Porphyromonas gingivalis, Actinobacillus actinomycetemcomitans, Fusobacterium nucleatum) no líquido amniótico de ovelhas prenhas. Puderam confirmar a patogenicidade destas bactérias, demonstrando que a maioria dos fetos foi a óbito. Apenas seis fetos do total de 22 infectados com os lipopolissacarídeos da bactéria Porphyromonas gingivalis sobreviveram e, nestes casos, foi possível observar características de inflamação presentes no líquido amniótico e no sangue do cordão umbilical ${ }^{24}$.

Apesar do fato de estudos em ovelhas não necessariamente poderem ser transpostos com as mesmas evidências para seres humanos, os resultados da pesquisa devem ser considerados. Algumas dúvidas ainda permanecem: qual a quantidade de lipopolissacarídeos que teria capacidade de induzir alterações gestacionais em humanos? Esta quantidade seria produzida e disseminada a partir do periodonto infectado?

Algumas revisões sistemáticas da literatura têm sido realizadas, buscando encontrar evidências suficientes para confirmar estas suspeitas. A associação entre doença periodontal e risco de parto pré-termo/nascimento de baixo peso foi avaliada em uma metanálise em 2002. Segundo os autores, poucas pesquisas puderam ser incluídas na revisão, sendo uma do tipo coorte e duas do tipo caso-controle. Apesar de concluírem que a relação de risco existe, sugeriram mais pesquisas com metodologias semelhantes, principalmente no que se refere à classificação das alterações periodontais, que vem sendo muito questionada nas diversas pesquisas publicadas ${ }^{25}$.

Em outra revisão sistemática, os autores avaliaram se o controle da doença periodontal durante a gestação teria algum impacto sobre a iniciação ou progressão de complicações gestacionais. Após incluírem apenas 12 das 660 pesquisas encontradas na busca bibliográfica, concluíram que a doença periodontal pode ser um fator de risco para nascimento pré-termo e/ou de baixo peso e que são necessários mais estudos longitudinais e intervencionais, buscando responder se as associações poderiam ser causais. Estudos intervencionais preliminares sugerem que a terapêutica periodontal reduz o risco de complicações gestacionais ${ }^{26}$.

As possíveis evidências na relação entre doença periodontal e complicações na gestação foram avaliadas em uma revisão de 25 estudos (13 casos-controle, nove coortes e três pesquisas clínicas), sendo que 18 encontraram evidências da associação e sete não puderam confirmá-las. As três pesquisas clínicas sugeriram que o tratamento periodontal pode reduzir em $50 \%$ o nascimento de prematuros. Apesar destas conclusões, os autores também ressaltaram a necessidade de padronizações metodológicas nas pesquisas ${ }^{27}$.

Outros autores concluíram em uma metanálise que gestantes com doença periodontal têm 4,2 mais chances de desenvolver parto prematuro do que as gestantes saudáveis $(4,2 ; \text { IC } 95 \%=2,6 \text { a } 6,9 ; \mathrm{p}<0,005)^{28}$. No entanto, como a maioria das pesquisas encontradas foi do tipo caso-controle e coorte, os autores não acreditam estar comprovada a suspeita de que o tratamento periodontal reduz os riscos durante a gestação.

Ensaios clínicos controlados são estudos preferenciais para sanarem as dúvidas existentes. Algumas pesquisas com estas características têm sugerido que o tratamento periodontal poderia reduzir o risco de parto prematuro baseado na diminuição dos lipopolissacarídeos presentes na bolsa periodontal infeccionada ${ }^{29}$.

Outra pesquisa em que se avaliou o efeito do tratamento periodontal no nascimento de recémnascidos prematuros e/ou de baixo peso selecionou 870 gestantes com gengivite, entre 18 e 42 anos. Destas, 580 receberam tratamento até a $28^{a}$ semana de gestação e 290 após o nascimento. A incidência das complicações obstétricas citadas no grupo de tratamento foi menor que no grupo controle, mesmo após ajuste estatístico para outros fatores de risco, sugerindo que a alteração bucal seja um fator de risco independente para parto prematuro/recém-nascidos de baixo peso naquela população ${ }^{30}$. 
No entanto, outro estudo não confirmou esta evidência. As mulheres selecionadas tinham entre $13 \mathrm{e}$ 17 semanas de gestação. As gestantes do grupo experimental $(n=413)$ receberam tratamento antes da $21^{\mathrm{a}}$ semana, enquanto aquelas do grupo controle $(n=410)$, após o parto. Apesar de o tratamento melhorar as condições gengivais, não houve diferença estatística entre os grupos estudados no que se refere à época de nascimento e peso do recém-nascido ${ }^{20}$.

Há grande heterogeneidade nos estudos disponíveis para aferir a relação dos métodos de mensuração da doença periodontal e os desfechos indesejáveis da gestação, não sendo possível realizar metanálise. Em 36 pesquisas selecionadas, foram utilizadas 13 diferentes definições para a doença periodontal. Pelas limitações metodológicas das pesquisas, os autores não puderam afirmar adequadas conclusões sobre o real efeito da doença periodontal nos desfechos da gestação ${ }^{31}$.

\section{Pré-eclâmpsia}

A pré-eclâmpsia acomete 5 a $8 \%$ das gestantes dos países em desenvolvimento e está relacionada à alta morbimortalidade materna e fetal ${ }^{32}$. Gestantes que desenvolvem pré-eclâmpsia têm maior risco de morte se comparadas com as pacientes sem esta condição ${ }^{33}$. Por outro lado, o nascimento de recém-nascidos prematuros e/ou de baixo peso em decorrência de condições mórbidas maternas pode causar prejuízos permanentes nas crianças, tais como: asma $^{34}$, alterações visuais, motoras e cognitivas ${ }^{35}$, dentre outras.

Apesar de a etiologia da pré-eclâmpsia ainda permanecer parcialmente desconhecida, relacionam-se alguns fatores de risco, tais como: primiparidade, obesidade, alterações renais, dentre outras. No entanto, nos últimos anos, tem-se estudado a influência das infecções na pré-eclâmpsia, incluindo-se como possível sua relação com a doença periodontal ${ }^{16}$.

Poucos estudos publicados relacionaram o risco entre periodontite e pré-eclâmpsia, mas a maioria encontrou aumento do risco relativo de desenvolvimento de pré-eclâmpsia em gestantes com doença periodon$\mathrm{tal}^{15-19}$. Mais recentemente, outros autores conduziram estudo tipo caso-controle, em que associaram a doença periodontal com a pré-eclâmpsia, apresentando razão de chances de $4,1^{36}$ e $7,9^{37}$.

Por outro lado, em pesquisa reunindo $345 \mathrm{pa}-$ cientes ( 115 casos e 230 controles) não se encontrou sustentação para a relação de risco, sugerindo que as outras pesquisas talvez tenham falhas metodológicas, como tamanho amostral inadequado e análises estatísticas inapropriadas ${ }^{38}$.
As explicações para esta relação poderiam se basear, por exemplo, nas lesões endoteliais freqüentemente encontradas em gestantes com pré-eclâmpsia. Sabe-se que algumas citocinas inflamatórias, principalmente o TNF-alfa, têm capacidade de lesar o endotélio vascular $^{39}$, sendo este fator uma das citocinas presentes e disseminadas na doença periodontal.

Outras pesquisas encontraram patógenos periodontais em placas de ateroma após análises laboratoriais ${ }^{40}$, sugerindo relação da doença periodontal com aterosclerose, sendo sugerida também a similaridade entre as lesões vasculares placentárias e as lesões ateroscleróticas ${ }^{18}$.

Após avaliação da presença de bactérias periodontopatogênicas em placentas humanas, observou-se que $50 \%$ daquelas pertencentes a gestantes com préeclâmpsia tiveram colonização positiva para Actinobacillus actinomycetemcomitans, Fusobacterium nucleatum ssp., Porphyromonas gingivalis, Prevotella intermedia, Tannerella forsythensis e Treponema denticola, enquanto no grupo controle (sem pré-eclâmpsia), as bactérias foram encontradas em apenas 14,3\% das placentas, sendo estas diferenças estatisticamente significantes ${ }^{41}$. Esta parece ser uma evidência importante a favor da disseminação hematogênica de bactérias bucais. Interessantemente, não se descreve associação com infecção amniótica e prematuridade neste estudo, que poderia ser uma correlação mais plausível do ponto de vista biológico do que com pré-eclâmpsia.

Deve-se lembrar que gestantes com pré-eclâmpsia, independente de afecções bucais, apresentam aumento dos níveis plasmáticos de algumas citocinas, tais como: IL-6 ${ }^{42}$, IL- $^{43}$, TNF-alfa ${ }^{44}$ e IL-10 ${ }^{43}$. Este aumento seria decorrente de um estado inflamatório generalizado, embora a significância destas alterações na patogênese da pré-eclâmpsia ainda permaneça obscura ${ }^{43}$.

Alguns autores analisaram a relação entre doença periodontal e pré-eclâmpsia em revisão sistemática e, apesar de encontrarem associação entre as duas afecções, os autores sugerem mais pesquisas neste sentido, já que, até o momento, a literatura é escassa ${ }^{27}$.

A relação de risco entre infecção periodontal e complicações gestacionais tem merecido atenção na atualidade. Há muitos resultados já publicados, em que os próprios autores discutem as limitações de suas conclusões. É possível que esta situação decorra da complexidade das complicações obstétricas, com as quais se encontraram ou se buscam associações com a doença periodontal. A pré-eclâmpsia, por exemplo, é uma alteração que apresenta etiologia ainda desconhecida, com diversos fatores de risco.

O papel das citocinas, principal elo entre doença periodontal e complicações gestacionais, também 
não foi totalmente desvendado. Não se pode esquecer que o principal fator a ser estudado no momento é a produção ou mesmo disseminação destas citocinas do sítio periodontal para o sangue da gestante. Apesar da dificuldade em se realizarem alguns tipos de estudos que possam suportar com credibilidade as evidências das associações suspeitas, é obrigatório que se desenhem novas pesquisas que avaliem a concentração exata das citocinas capazes de causar complicações obstétricas e determinar se esta quantidade pode ser produzida e disseminada pelo periodonto durante a vigência da infecção. Novos estudos clínicos que realizem tratamento em gestantes com infecção periodontal também auxiliariam na correlação da diminuição da taxa de complicações obstétricas e a relação com este tratamento. Os conceitos atuais apontam para etiologia multifatorial, tanto da prematuridade quanto da pré-eclâmpsia, e a doença periodontal pode ser um aspecto importante para mulheres com determinado tipo de resposta imunológica. O estudo da interação genético-ambiental é um caminho interessante para elucidar a conexão possível entre inflamação, préeclâmpsia e prematuridade.

Embora a literatura disponível atualmente não permita concluir efetivamente que infecções bucais podem influenciar na ocorrência de complicações obstétricas, a atenção com a saúde bucal da gestante não poderá ser dispensada durante os cuidados pré-natais e, se possível, pré-concepcionais. Deve-se recomendar que todas as mulheres grávidas atentem para sua saúde bucal, com os cuidados de higiene, pesquisando sistematicamente sangramento gengival, dor e mobilidade dentária. A avaliação odontológica periódica da gestante pode permitir que o cuidado com a saúde dentária seja mais efetivo em prevenir eventuais repercussões de afecções bucais sobre sua saúde como um todo.

\section{Referências}

1. Raber-Durlacher JE, van Steenbergen TJ, van der Velden U, de Graaff J, Abraham-Inpijn L. Experimental gingivitis during pregnancy and post-partum: clinical, endocrinological, and microbiological aspects. J Clin Periodontol. 1994;21 (8):549-58.

2. Loee H, Silness J. Periodontal disease in pregnancy. I. Prevalence and severity. Acta Odontol Scand. 1963;21:533-51.

3. Hugoson A. Gingivitis in pregnant women. A longitudinal clinical study. Odontol Revy. 1971;22(1):65-84.

4. Mascarenhas P, Gapski R, Al-Shammari K, Wang HL. Influence of sex hormones on the periodontium. J Clin Periodontol. 2003;30(8):671-81.

5. Nyman S, Lindhe J. Exames em pacientes com doença periodontal. In: Lindhe J, editor. Tratado de periodontia clínica. Rio de Janeiro: Guanabara Koogan; 1999. p. 271-80.

6. Beck JD, Offenbacher S. Systemic effects of periodontitis: epidemiology of periodontal disease and cardiovascular disease. J Periodontol. 2005;76(1 1 Suppl):2089-100.

7. Hofling JF, Gonçalves RB, Kamiya RU. Histórico e introdução à imunologia. In: Hofling JF, Gonçalves RB, editores. Imunologia para odontologia. Porto Alegre: Artmed; 2006. p. 13-28.

8. Konopka T, Rutkowska M, Hirnle L, Kopec W. [IL-1 beta and PGE2 production in whole blood and gingival fluid in women with periodontitis and preterm low birth weight]. Ginekol Pol. 2004;75(5):352-60. Polish.

9. Erdemir EO, Duran I, Haliloglu S. Effects of smoking on clinical parameters and the gingival crevicular fluid levels of IL-6 and TNF-alpha in patients with chronic periodontitis. J Clin Periodontol. 2004;31 (2):99-104.

10. Yang ML, Chen YH, Chen TC, Lin WR, Lin CY, Lu PL. Case report: infective endocarditis caused by Brevundimonas vesicularis. BMC Infect Dis. 2006;6:179.

11. Ide M, Jagdev D, Coward PY, Crook M, Barclay GR, Wilson RF. The short-term effects of treatment of chronic periodontitis on circulating levels of endotoxin, C-reactive protein, tumor necrosis factor-alpha, and interleukin-6. J Periodontol. 2004;75(3):420-8.
12. Promsudthi A, Pimapansri S, Deerochanawong C, Kanchanavasita $W$. The effect of periodontal therapy on uncontrolled type 2 diabetes mellitus in older subjects. Oral Dis. 2005; 11 (5):293-8.

13. Offenbacher S, Boggess KA, Murtha AP, Jared HL, Lieff S, McKaig $R G$, et al. Progressive periodontal disease and risk of very preterm delivery. Obstet Gynecol. 2006; 107(1):29-36.

14. Offenbacher S, Katz V, Fertik G, Collins J, Boyd D, Maynor G, et al. Periodontal infection as a possible risk factor for preterm low birth weight. J Periodontol. 1996;67(10 Suppl):1 103-13.

15. Boggess KA, Lieff S, Murtha AP, Moss K, Beek J, Offenbacher S. Maternal periodontal disease is associated with an increased risk for preeclampsia. Obstet Gynecol. 2003;101(2):227-31.

16. Canakci V, Canakci CF, Canakci H, Canakci E, Cicek Y, Ingec $M$, et al. Periodontal disease as a risk factor for pre-eclampsia: a case control study. Aust N Z J Obstet Gynaecol. 2004;44(6): 568-73.

17. Contreras A, Herrera JA, Soto JE, Arce RM, Jaramillo A, Botero JE. Periodontitis is associated with preeclampsia in pregnant women. J Periodontol. 2006;77(2):182-8.

18. Oettinger-Barak $O$, Barak $S$, Ohel $G$, Oettinger $M$, Kreutzer $H$, Peled $M$, et al. Severe pregnancy complication (preeclampsia) is associated with greater periodontal destruction. J Periodontol. 2005;76(1):134-7.

19. Riche EL, Boggess KA, Lieff S, Murtha AP, Auten RL, Beck JD, et al. Periodontal disease increases the risk of preterm delivery among preeclamptic women. Ann Periodontol. 2002;7(1): 95-101.

20. Michalowicz BS, Hodges JS, DiAngelis AJ, Lupo VR, Novak M, Ferguson JE, et al. Treatment of periodontal disease and the risk of preterm birth. N Engl J Med. 2006;355(18): 1885-94.

21. Perroni AG, Bittar RE, Zugaib M. Corioamnionite como causa de trabalho de parto prematuro espontâneo. Femina. 2006;34(1):67-71. 
22. Nomura ML, Passini Junior, Oliveira UM. Group B streptococcus colonization in preterm labor and preterm premature rupture of membranes. Int J Gynaecol Obstet. 2005;91(1):69-70.

23. Teles $E P B$, Faúndes $A$, Barini $R$, Passini Júnior R. Fatores de risco para parto prematuro numa amostra de gestantes brasileiras: I. Fatores pré-gestacionais. Rev Bras Ginecol Obstet. 1992;14(4):161-4.

24. Newnham JP, Shub A, Jobe AH, Bird PS, Ikegami M, Nitsos I, et al. The effects of intra-amniotic injection of periodontopathic lipopolysaccharides in sheep. Am J Obstet Gynecol. 2005; 193(2):313-21.

25. Madianos PN, Bobetsis GA, Kinane DF. Is periodontitis associated with an increased risk of coronary heart disease and preterm and/or low birth weight births? J Clin Periodontol. 2002;29 Suppl 3:22-36.

26. Scannapieco FA, Bush RB, Paju S. Periodontal disease as a risk factor for adverse pregnancy outcomes. A systematic review. Ann Periodontol. 2003;8(1):70-8.

27. Xiong X, Buekens P, Fraser WD, Beck J, Offenbacher S. Periodontal disease and adverse pregnancy outcomes: a systematic review. BJOG. 2006; $113(2): 135-43$.

28. Khader YS, Ta'ani Q. Periodontal diseases and the risk of preterm birth and low birth weight: a meta-analysis. J Periodontol. 2005;76(2): 161-5.

29. Shub A, Swain JR, Newnham JP. Periodontal disease and adverse pregnancy outcomes. J Matern Fetal Neonatal Med. 2006; 19(9):521-8.

30. Lopez NJ, Da Silva I, Ipinza J, Gutierrez J. Periodontal therapy reduces the rate of preterm low birth weight in women with pregnancy-associated gingivitis. J Periodontol. 2005;76/11 Suppl):2144-53

31. Vettore MV, Lamarca GA, Leão ATT, Thomaz FB, Sheiham A, Leal MC. Infecção periodontal e desfechos indesejáveis da gestação: uma revisão sistemática dos estudos epidemiológicos. Cad Saúde Pública. 2006;22(10):2041-53.

32. Report of the National High Blood Pressure Education Program Working Group on High Blood Pressure in Pregnancy. Am J Obstet Gynecol. 2000;183(1):S1-S22.

33. Irgens HU, Reisaeter L, Irgens LM, Lie RT. Long term mortality of mothers and fathers after pre-eclampsia: population based cohort study. BM. 2001;323(7323):1213-7.
34. Chatkin MN, Menezes AM. The association between low birthweight and asthma: a systematic literature review. Rev Panam Salud Publica. 2005;17(2):102-9.

35. Graziano RM, Leone CR. Frequent ophthalmologic problems and visual development of extremely preterm newborn infants. J Pediatr (Rio J). 2005;81 (1 suppl):S95-100.

36. Cota LO, Guimarães AN, Costa JE, Lorentz TC, Costa FO. Association between maternal periodontitis and an increased risk of preeclampsia. J Periodontol. 2006;77(12):2063-9.

37. Kunnen A, Blaauw J, van Doormaal JJ, van Pampus MG, van der Schans CP, Aarnoudse JG, et al. Women with a recent history of early-onset pre-eclampsia have a worse periodontal condition. J Clin Periodontol. 2007;34(3):202-7.

38. Khader YS, Jibreal M, Al-Omiri M, Amarin Z. Lack of association between periodontal parameters and preeclampsia. J Periodontol. 2006;77(10): 1681-7.

39. Daher S, Mattar R, Sass N. Doença hipertensiva específica da gravidez: aspectos imunológicos. In: Sass N, Camano L, Moron $A F$, editores. Hipertensão arterial e nefropatias na gravidez. Rio de Janeiro: Guanabara Koogan; 2006. p. 45-56.

40. Haraszthy VI, Zambon JJ, Trevisan M, Zeid M, Genco RJ. Identification of periodontal pathogens in atheromatous plaques. J Periodontol. 2000;71(10):1554-60.

41. Barak S, Oettinger-Barak O, Machtei EE, Sprecher H, Ohel G. Evidence of periopathogenicmicroorganismsinplacentasofwomen withpreeclampsia. J Periodontol. 2007;78(4):670-6.

42. Afshari JT, Ghomian N, Shameli A, Shakeri MT, Fahmidehkar MA, Mahajer E, et al. Determination of Interleukin-6 and Tumor Necrosis Factor-alpha concentrations in Iranian-Khorasanian patients with preeclampsia. BMC Pregnancy Childbirth. 2005;5:14.

43. Madazli R, Aydin S, Uludag S, Vildan O, Tolun N. Maternal plasma levels of cytokines in normal and preeclamptic pregnancies and their relationship with diastolic blood pressure and fibronectin levels. Acta Obstet Gynecol Scand. 2003;82(9):797-802.

44. Hayashi M, Ueda Y, Yamaguchi T, Sohma R, Shibazaki M, Ohkura $T$, et al. Tumor necrosis factor-alpha in the placenta is not elevated in pre-eclamptic patients despite its elevation in peripheral blood. Am J Reprod Immunol. 2005;53(3): 1 13-9. 